\title{
Production of a high-efficiency cellulase complex via $\beta$-glucosidase engineering in Penicillium oxalicum
}

\author{
Guangshan Yao ${ }^{1 \dagger}$, Ruimei Wu ${ }^{1 \dagger}$, Qinbiao Kan ${ }^{1 \dagger}$, Liwei Gao ${ }^{1}$, Meng Liu', Piao Yang ${ }^{1}$, Jian Du ${ }^{1}$, Zhonghai Li $3^{*}$
} and Yinbo Qu ${ }^{1,2^{*}}$ (D)

\begin{abstract}
Background: Trichoderma reesei is a widely used model cellulolytic fungus, supplying a highly effective cellulase production system. Recently, the biofuel industry discovered filamentous fungi from the Penicillium genus as a promising alternative to T. reesei.
\end{abstract}

Results: In our study, we present a systematic over-expression analysis of nine $\beta$-glucosidase encoding genes in the wild-type strain 114-2 of Penicillium oxalicum. We found that the over-expression of BGL1, BGL4, or BGL5 significantly enhanced both $\beta$-glucosidase activity and hydrolysis efficiency of the enzyme system on filter paper. We utilised two strategies to over-express $\beta$-glucosidase in the strain RE-10 that-although over-producing cellulase, does so at the cost of the cellulase mixture deficiency. The constitutive promoter of gene pde_02864 encoding 405 ribosomal protein 58 was used to over-express three $\beta$-glucosidases: BGL1, BGL4, and BGL5. We found that all mutants show significantly enhanced levels of $\beta$-glucosidase at transcriptional, protein, and activity levels. Furthermore, the inducible promoter from bg/2 was used to conditionally over-express the $\beta$-glucosidases BGL1 and BGL4. Surprisingly, this induced expression strategy enables significantly improved expression efficiency. The BGL1 over-expressing mutant 11-13 particularly improved the $\beta$-glucosidase activity at a factor of 65 -folds, resulting in levels of up to $150 \mathrm{U} / \mathrm{ml}$. All our BGL over-expression mutants displayed significant enhancement of cellulolytic ability on both microcrystalline cellulose and filter paper. In addition, they substantially reduced the enzyme loads in the saccharification of a natural lignocellulose material delignified corncob residue (DCCR). The mutant 14-32 with over-expression of BGL4 achieved the highest glucose yield in the saccharification of DCCR at only $25 \%$ enzyme load compared to the parental strain RE-10.

Conclusions: In summary, genetically engineering P. oxalicum to significantly improve $\beta$-glucosidase activity is a potent strategy to substantially boost the hydrolytic efficiency of the cellulase cocktail, which will ultimately lead to a considerable reduction of cost for biomass-based biofuel.

Keywords: Penicillium oxalicum, Biofuel, $\beta$-glucosidase, Genetic engineering

\footnotetext{
*Correspondence: Izhlzh@vip.126.com; quyinbo@sdu.edu.cn

${ }^{\dagger}$ Guangshan Yao, Ruimei Wu and Qinbiao Kan contributed equally to this work

${ }^{2}$ National Glycoengineering Research Center, Shandong University, Jinan

City 250100, Shandong Province, China

${ }^{3}$ Department of Bioengineering, Qilu University of Technology, Jinan

City 250353, Shandong Province, China

Full list of author information is available at the end of the article
} 


\section{Background}

The conversion of plant-based biomass into biofuel and chemicals is a promising course to absorb the upcoming shortage of fossil fuels without any serious environmental problems. Cellulose is the major building block of plant biomass [1]. Fungal cellulases transform plant polysaccharides into fermentable sugars, which in turn determine the cost of plant-based biofuel. Throughout the enzyme industry, the champion for the production of cellulase cocktail is Trichoderma reesei [2-4]. However, an increasing number of publications demonstrate better hydrolysing lignocellulose performance of the cellulase enzyme mixture obtained from Penicillium strains. Thus, Penicillium strains are considered a potent alternative to the T. reesei cellulase cocktail [5].

Three Penicillium oxalicum strains in particular, secrete a more balanced lignocellulose-degrading enzyme system and display higher $\beta$-glucosidase activity compared to T. reesei. These are: P. oxalicum 114-2 [6], P. oxalicum GZ-2 [7], and P. oxalicum 16 [8]. P. oxalicum 114-2 has been studied in our laboratory for more than 30 years. Multiple cycles of mutagenesis and screening led to the carbon catabolite repression-resistant mutant JU-A10-T with a volume productivity of $160 \mathrm{IU} / \mathrm{Lh}$. For the last 20 years, this mutant produces cellulase enzyme preparations at industrial scale in China [9]. Recently, its genome has been sequenced, providing valuable information to mine novel components that play key roles in plant cell wall deconstruction [6]. Furthermore, the signal transduction, inducer transportation, and transcription regulation mechanism of the cellulase gene expression of $P$. oxalicum has been partially uncovered. The sequenced genome, high-efficient gene knock-out techniques, and system biology (RNA-seq and proteomics) are the methods that have led to this advance. Gene disruption analysis allowed the identification of the three major cellodextrin transporters $\mathrm{CdtC}, \mathrm{CdtD}$, and $\mathrm{CdtG}$ that play crucial roles in cellulase induction [10]. Hu et al. [11] revealed that the G protein-cAMP signaling pathway downregulates the expression of cellulolytic genes. Furthermore, a single-gene deletion library has been established for 470 transcription factors and 15 novel and 4 major transcriptional regulators. Their roles in the cellulase expression regulatory network have been identified and characterized [12]. The Reconstruction of Expression Regulatory Network (REXRN) technology has been developed as a new strategy to engineer fungi that enhance cellulase and protein production. One mutant in particular (RE-10 from REXRN) displayed a drastic increase in cellulase and hemicellulase production, and produced even higher values compared to the industrial strain JU-A10-T [13]. However, the $\beta$-glucosidase of RE-10 has not yet been improved to the same level as other inducible cellulases. This makes further improvement of its $\beta$-glucosidase activity necessary.

Beta-glucosidase is the rate-limiting enzyme because it is responsible for the final step of lignocellulose hydrolysis and converts cellobiose as well as short cellodextrins into glucose [14]. Fungal $\beta$-glucosidases are classified into glycosyl hydrolases families 3 and 1 (GH3 and GH1) [15, 16]. Different proteins exhibit various characteristics in specific activity, substrate specificity, stability and others in the same family of $\beta$-glucosidase [17]. The genome of P. oxalicum includes $11 \beta$-glucosidase encoding genes, of which only $\beta$-glucosidase 1 (BGL1) and $\beta$-glucosidase 2 (BGL2) have been identified $[18,19]$.

To solve the shortage of $\beta$-glucosidase in T. reesei enzyme cocktails, a time-consuming and laborious process is used to supplement heterologous $\beta$-glucosidases from Aspergillus into commercial cellulase preparations [14]. We systemically screened the $\beta$-glucosidases via over-expression analysis and found three $\beta$-glucosidases from $P$. oxalicum to have a promising enzymatic performance. The three BGLs were over-expressed either constitutively, or inductively by means of two different types of promoters. We obtained a number of high-yield $\beta$-glucosidase producers and their $\beta$-glucosidase yields were elevated from twofold to 65 -fold. Microcrystalline cellulose, filter paper and corncob residue were used as substrates to assess hydrolysis efficiency of the enzyme complex.

\section{Results and discussion}

Systematic over-expression analysis of nine $\beta$-glucosidases in P. oxalicum 114-2

The $\beta$-glucosidases of $P$. oxalicum are encoded in a total of 11 genes. Among these, four $\beta$-glucosidases belong to the glucoside hydrolase family 1 (GH1) with the other seven BGLs belonging to GH3. Using multiple algorithms, we found the signal peptides for five $\beta$-glucosidases (Table 1 ), implying a high likelihood for these enzymes to secret extracellularly. However, of these, only BGL1 had been detected in a previous secretome analysis [6]. In order to systematically examine all $\beta$-glucosidases in P. oxalicum, we performed a systematic over-expression analysis, using a constitutive promoter from gene pde_02864 to drive BGL expression. We obtained 9 out of 11 BGLs overexpression transformants, while over-expression mutants for the remaining two genes were not available after several rounds of genetic transformation. The promoter of gene pde_02864 that decodes the 40S ribosomal protein S8, has previously been identified as a strong and constitutively promoter [12]. We then evaluated the $p$ NPGase activities and filter paper hydrolysis rate of all the obtained $\beta$-glucosidase over-expression mutants (obtaining at least two transformants for each gene). Both the BGL(X) 
Table 1 Systematic screening the major $\beta$-glucosidases

\begin{tabular}{|c|c|c|c|c|c|c|}
\hline $\mathrm{BG}(\mathrm{X})$ & Gene ID & $\begin{array}{l}\text { Signal } \\
\text { peptide }\end{array}$ & GH family & $\begin{array}{l}\text { Fold change } \\
\text { (transcript level) }\end{array}$ & $\begin{array}{l}\text { Fold change } \\
\text { ( } p \text { NPGase activity) }\end{array}$ & $\begin{array}{l}\text { Fold change } \\
\text { (filter paper } \\
\text { hydrolysis rate) }\end{array}$ \\
\hline BGL1 & PDE_02736 & Yes & $\mathrm{GH} 3$ & 3.93 & 2.023 & 1.37 \\
\hline BGL2 & PDE_00579 & No & $\mathrm{GH} 1$ & 4.48 & 1.537 & 0.68 \\
\hline BGL3 & PDE_01277 & Yes & $\mathrm{GH} 1$ & 40.4 & 0.650 & 0.92 \\
\hline BGL4 & PDE_01565 & Yes & $\mathrm{GH} 3$ & 492.1 & 9.335 & 1.20 \\
\hline BGL5 & PDE_02905 & Yes & $\mathrm{GH} 3$ & 132.0 & 6.439 & 1.10 \\
\hline BGL6 & PDE_09019 & Yes & $\mathrm{GH3}$ & 17.8 & 1.797 & 0.89 \\
\hline BGL7 & PDE_02108 & No & $\mathrm{GH} 3$ & 79.6 & 2.427 & 0.73 \\
\hline BGL8 & PDE_04859 & No & $\mathrm{GH} 1$ & 68.0 & 0.868 & 0.89 \\
\hline BGL9 & PDE_03485 & No & $\mathrm{GH} 3$ & 13.2 & 0.868 & 0.90 \\
\hline
\end{tabular}

Yes with signal peptides, No without signal peptides

over-expression mutants and RE-10 were cultured in cellulose medium for enzyme production. Our results (Table 1) show, that the over-expression of seven BGLs significantly increased $p$ NPG activity. Among these, overexpression of BGL1, BGL4, BGL5, and BGL7 resulted in more than twice $\beta$-glucosidase activities of that of WT. In parallel, the crude enzyme from BGL1, BGL4, and BGL5 over-expression mutants significantly improved the hydrolysis rate of filter paper (see Table 1). However, over-expression of both $b g l 3$ and $b g l 8$ slightly reduced the extracellular $\beta$-glucosidase (less than twofold) and FPase activities. BGL8 is an intracellular $\beta$-glucosidase and thus hydrolyze intracellular cellobiose into glucose, which enhancing the repression and alleviating the induction of cellulolytic system. BGL3 had been expressed and purified in Pichia pastoris. However, the BGL3 did not showed activities against both $p$ NPG and salicin in vitro (data not shown), and their roles (BGL3 and BGL8) in regulation of the expression of cellulase and $\beta$-glucosidase required further investigation. Therefore, BGL1, BGL4, and BGL5 are considered as the most efficient $\beta$-glucosidases and hence, we targeted them in the following strain engineering for a cellulase high-producer.

Similar to previous cellodextrin transporter studies [10], gene knockout analysis is unhelpful for identification of the essential genes that are responsible for $\beta$-glucosidase activity, due to high functional redundancy [20]. We propose that the single-gene over-expression analysis we establish in this study is effective to discriminate the important $\beta$-glucosidases, and other highredundant genes (or gene families) in filamentous fungi.

\section{Sequence and phylogenetic analysis of three major $\beta$-glucosidases from $P$. oxalicum and other cellulolytic fungi}

BGL1 was the most conserved extracellular $\beta$-glucosidase, and its homolog could be found in many cellulolytic fungi, such as $P$. brasilianum, $T$. reesei, Neurospora crassa, Aspergillus nidulans, A. fumigatus, A. niger and Talaromyces cellulolyticus, and plant pathogens with a capacity of degrading plant cell wall, including Botrytis cinerea, Magnaporthe oryzae (Fig. 1), implying its essential role in lignocellulose deconstruction in the natural system. Orthologs of BGL5 can be found in most of the above cellulolytic fungi, with the exception of $T$. reesei and $N$. crassa (Fig. 1). Interestingly, BGL4 could only be identified in P. oxalicum and the Fusarium genus and forms a clade separate from BGL1 and BGL5 (Fig. 1). This suggests an evolutionary development from gene expansion. However, most of the $\beta$-glucosidases from the Fusarium genus remain unidentified.

All three efficient $\beta$-glucosidases are classified as members of the GH3 family (Table 1), and have three functional domains: the $\mathrm{N}$-terminal domain, the $\mathrm{C}$-terminal domain, and a fibronectin-like domain with unknown function (see Additional file 1: Figure S1). According to a previous study, the former two domains are likely forming a catalytic pocket [21].

\section{Over-expression of $\beta$-glucosidases in RE-10 using a constitutive promoter}

The cellulase over-producer RE-10 has previously been obtained by redesigning the regulatory pathway of cellulase gene expression in P. oxalicum. As a result, most enzyme proteins and their corresponding activities, were significantly enhanced, with the exception of BGL1 and $p$ NPGase activity remaining unaffected [13]. Therefore, improvement of the $\beta$-glucosidase activity in RE-10 was the main goal of our study. Via systematic screening, we identified BGL1, BGL4, and BGL5 as the most promising $\beta$-glucosidases for industrial scale Penicillium cellulase production.

The three $\beta$-glucosidases encoding genes pde_02736, pde_01565 and pde_02905, were driven by the promoter 


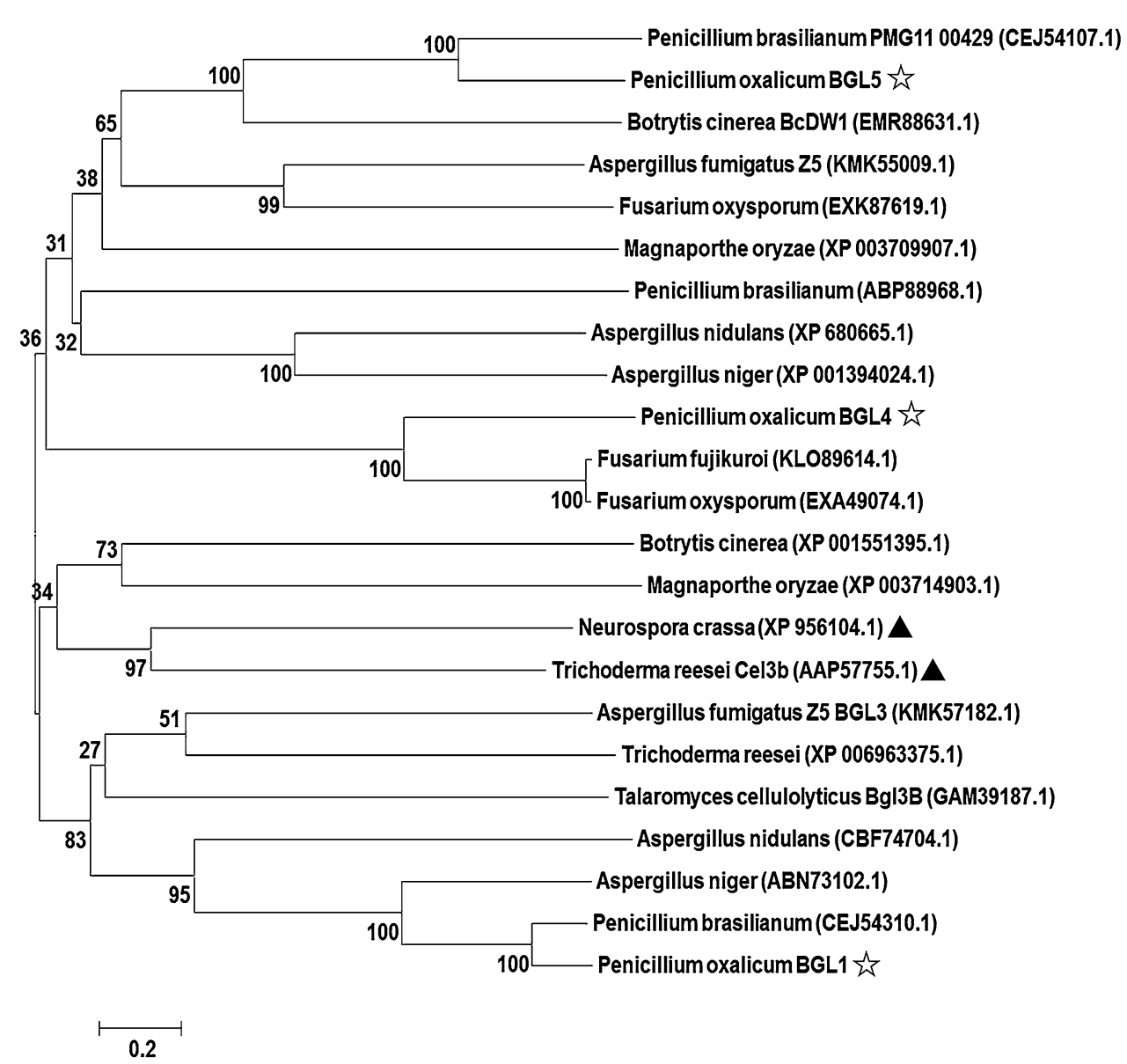

Fig. 1 Phylogenetic analysis of BGL1, BGL4, and BGL5 proteins in P. oxalicum and their orthologs from other filamentous fungi. Construction of phylogenetic tree was based on neighbor-joining analysis and full-length amino acids for all proteins were used to generate the tree with the software MEGA 6.0. Filled triangle indicates the characterized $B G L(X)$ homolog in $N$. crassa or T. reesei. Star indicates that the $B G L(X)$ from $P$. oxalicum

of pde_02864 and expressed in the background of RE-10. Following single spore isolation, three BGL1, two BGL4, and two BGL5 over-expression mutants were selected for further analysis. All the putative over-expression mutants showed increased $p$ NPGase activity. As shown in Fig. 2, all the BGL over-expression mutants also had a significant increase $(p \leq 0.01)$ in $\beta$-glucosidase activity. Three BGL1 over-expression mutants increased $p$ NPGase activity over 10,12 , fivefold, respectively, compared to the parental strain RE-10. Remarkably, the $p$ NPGase of one of them (C1-57) reached up to $30 \mathrm{IU} / \mathrm{ml}$, which is almost the highest value reported throughout all Penicillium strains with the ability for lignocellulose degradation. This possibly correlates with the fact that BGL1 has a lower $\mathrm{Km}$ than the BGLs from other fungi [18]. In addition, both BGL4 and BGL5 over-expression mutants displayed twofold higher $\beta$-glucosidase activity over RE-10 at 120 and $144 \mathrm{~h}$. Cellobiose is proposed as the natural substrate of $\beta$-glucosidase and is the product of $\mathrm{CBH}$ and EG during cellulose hydrolysis. To further illuminate their enzymatic performance of the BGL(X) over-expression mutants, we examined their cellobiose hydrolysis activity, and found that all the BGL(X) overexpression mutants improved glucose release from cellobiose by more than tenfold, which is consistent with the above $p$ NPGase activity analysis (see Additional file 2: Figure S2).

Inducible over-expression $\beta$-glucosidases in RE-10 Intracellular $\beta$-glucosidase BGL2 in P. oxalicum has previously been identified as a negative regulator for cellulase gene expression [19]. Interestingly, its expression pattern is similar to other inducible cellulase genes, such cel7A-2, cel5B and cel7B. As a result, the bgl2 promoter was designed to over-express secretory $\beta$-glucosidases. Multiple rounds of transformation and screening led to identification of three mutants for BGL1 over-expression (I1-2, I1-3, and I1-4), and two mutants for BGL4 


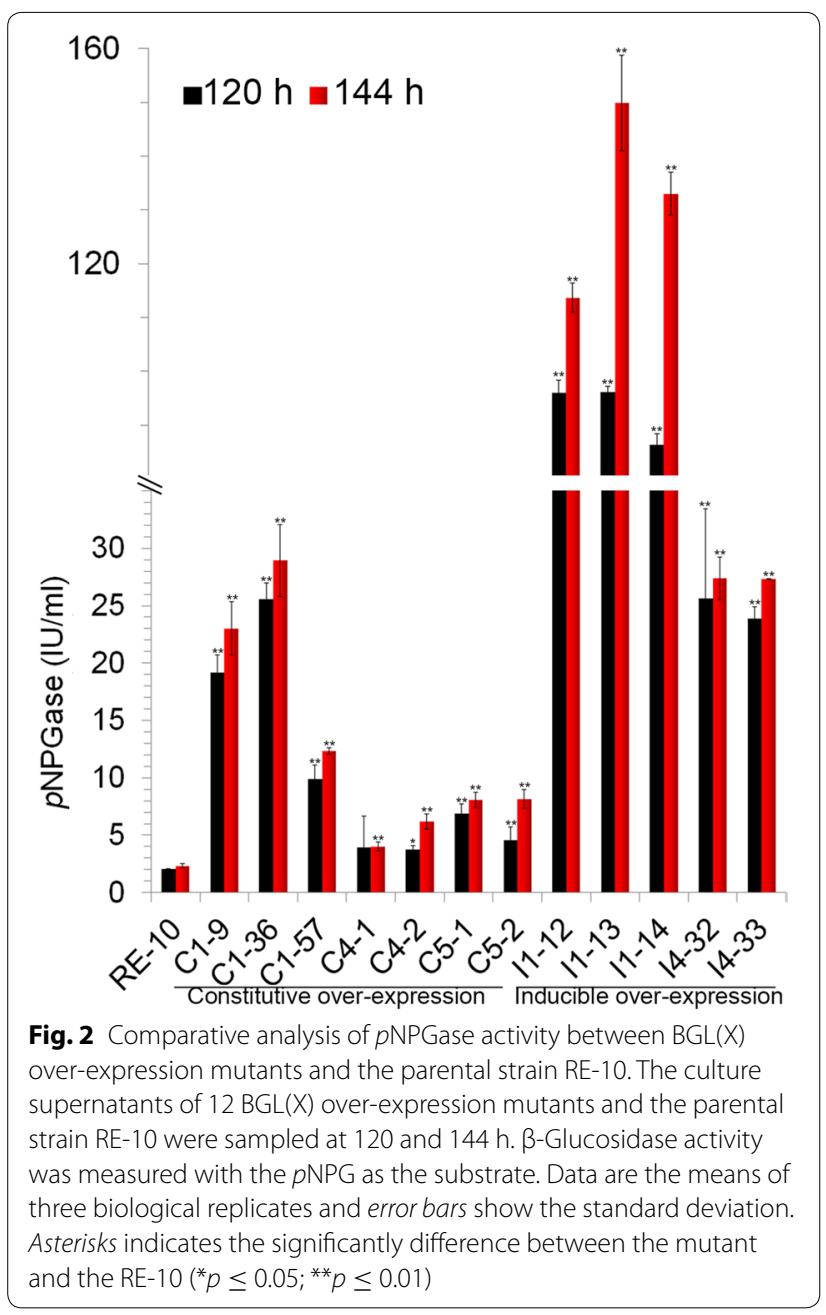

over-expression (I4-32 and I4-33). Despite considerable effort, no sur-resistant transformants for BGL5 could be obtained. Notably, all inducible mutants (I1-12, I1-13, I1-14, I4-32, and I4-33) displayed drastic increases in $\beta$-glucosidase activity towards both $p$ NPG and cellobiose, compared to their respective constitutive overexpression mutants. One of them in particular (I1-13) has a $p$ NPGase activity of $150 \mathrm{U} / \mathrm{ml}$ (Fig. 2) and a cellobiose activity of $36 \mathrm{U} / \mathrm{ml}$ (see Additional file 2: Figure $\mathrm{S} 2$ ). These activities are more than 65- and 93-fold higher than those of RE-10. In $T$. reesei, the strong $c b h 1$ promoter was previously used to drive bgll expression, but the recombinant strain produced a $p$ NPGase activity of only about $30 \mathrm{U} / \mathrm{ml}$ [22], which is significantly lower compared to that found in our study.

Genetic engineering of the regulatory machinery of cellulolytic genes is a promising strategy to create protein over-producers. Cellobiohydrolase, endo-glucanase, and other proteins that play significant roles in cellulose degradation, were all synchronously improved in our previous work, except for $\beta$-glucosidase [13]. Another pathway-specific regulatory mechanism for the expression of $\beta$-glucosidase is present in cellulolytic fungi and the $\beta$-glucosidase specific regulator BglR, which was found in $T$. reesei further strengthened this proposal [23]. As a consequence, additional improvement of $\beta$-glucosidase levels in the engineered cellulase over-producers is crucial for producing efficient as well as more balanced enzyme mixtures.

A recent study reports a strong cellulose induction of the gene $b g l 2$ and corresponding repression by glucosemediated carbon catabolite repression (CCR) [12]. The expression pattern of this gene is highly synchronous with cellulase gene induction. Furthermore, the cellulase gene transcription activator ClrB activates its level, while the repressor CreA represses its level. Interestingly, both the $b g l 2[20,24]$ and its regulators CreA/Cre1 $[25,26]$ are highly conserved in the cellulolytic fungi $N$. crassa and $T$. reesei. Our data further highlights the $b g l 2$ promoter to be a highly efficient and inducible promoter in $P$. oxalicum and likely also in other cellulolytic fungi, such as $N$. crassa and $T$. reesei. It will be a particularly valuable tool for strain genetic engineering, to conditionally superexpress other essential enzymes or proteins.

The sugars produced during the fermentation process were examined by high-performance liquid chromatography (HPLC). As expected, cellobiose was only detected in RE-10, but not in the other $\beta$-glucosidase over-expression mutants (see Additional file 3: Figure S3). In fact, the cellobiohydrolase and endo-glucanase activities were slightly enhanced in the $\beta$-glucosidase mutants (data not shown). The expression level of $\mathrm{CBH}$ and EG encoding genes were further analyzed (Additional file 4: Figure S4). The result showed that the expression levels of major $\mathrm{CBH}$ encoding gene $\operatorname{cbh} 1$ in $\mathrm{C} 1-9$, and major EG gene eg1 in C1-9, I1-12 and C4-1 were up-regulated significantly. On the other hand, previous assumptions pointed towards cellobiose to be the inhibitor of both cellobiohydrolase and endo-glucanase [27]. As a result, it was suggested that over-expression of $\beta$-glucosidase might indirectly increase both cellobiohydrolase and endo-glucanase activities by removing the product feedback inhibition caused by cellobiose.

\section{Improvement of microcrystalline cellulose degradation via over-expression of $\beta$-glucosidases in RE-10}

We assayed fungal hyphae extension rates of all mutants and used higher concentration cellulose in the plate medium to evaluate cellulolytic ability. Hyphae extension speeds and colony diameters of most of the BGL mutants resemble that of RE-10 (Fig. 3), which indicates that overexpression of BGLs did not affect fungal growth. However, the mutants C1-36 and C5-2 decreased fungal growth 
rate. A possible reason for this could lie in the genomic sites of expression cassette insertion, so we removed these two strains from further analysis. The cellulolytic zones of 8 over-expression strains increased significantly compared to RE-10, including C1-9, C1-36, C1-57, C5-1, I1-12, C4-1, I1-14, I4-32 and I4-33 (Fig. 3). This correlates with our results for the $\beta$-glucosidase activity assay. Our data demonstrates that over-expression of $\beta$-glucosidases significantly improves the fungal cellulolytic ability.

\section{Up-regulation of $B G L(X)$ in the mutants at both transcription and protein levels}

Although all the above mutants have been verified by PCR using specific primers (data not show), the transcript abundance of $\beta$-glucosidase genes has not been examined. Fluorescence quantitative polymerase chain reaction (qPCR) was performed to investigate the transcript abundance of BGL(X). The results are shown in Fig. 4. Both the C1-9 and I1-12 strains substantially upregulated their bgl1 expression levels in both glucose and cellulose conditions. Markedly, after cellulose induction for $4 \mathrm{~h}$, the transcriptional level of bgll in C1-9 increases about 1000-fold, and in I1-12 up-regulates 10,000-fold. The expression level of bgl5 in C5-1 was also greatly upregulated with values of up to 5000-fold in comparison to RE-10. The expression level of bgl4 in C4-1 and I4-32 was also significantly elevated. Our data confirmed that all of the $\beta$-glucosidase mutants we used, improved the corresponding $\beta$-glucosidase at the transcription level. Given that the expression levels of all three $b g l$ genes were comparable in their over-expression strains, we assumed that higher specific activity and substrate affinity of BGL1 contributes to higher $\beta$-glucosidase activity in the bgl1-over-expressing strains C1-9, C1-36, C1-57, I1-13, and I1-14. Furthermore, the SDS-PAGE and MS analysis confirmed that the BGL bands were significantly improved compared to those of RE-10 in the overexpression mutants (Fig. 5). The BGL1 amount of Il-12 in particular, is more abundant compared to that of $\mathrm{CBHI}$ (PDE_07945), which is the dominant protein in the cellulolytic secretome $[6,28,29]$.

The regulation model of the three $\beta$-glucosidases was analyzed based on three kinds of different promoterdriven expression patterns. Three different promoters were from gene $b g l(\mathrm{x}), p d e \_02864$ and $b g l 2$, respectively. The expression pattern of BGL1 and BGL4 was similar: low-level expression under glucose and high-level expression when induced by cellulose (Fig. 4 and Additional file 5: Figure S5), whose regulation model resemble $\mathrm{CBH}$ and EG. However, the $b g l 5$ displayed constitutive expression and independent of carbon source (Fig. 4c).

\section{Over-expressing $\beta$-glucosidases enhanced the filter paper hydrolysis}

For the process of lignocellulose hydrolysis, $\beta$-glucosidases affect the last step and are also the important rate-limiting enzymes. To further test the cellulolytic ability of the above $\beta$-glucosidase over-expression strains, filter paper was used as substrate. The result is shown in Fig. 6. All $\beta$-glucosidase mutants exhibit a significant increase in glucose release compared to RE-10. This suggests that high $\beta$-glucosidase activity has a strong impact on filter paper hydrolysis (Fig. 3). Most of $\beta$-glucosidase over-expression mutants increase the FPase activity over twofold compared to RE-10. This result further confirmed the significant role of $\beta$-glucosidase as rate-limiting enzyme in

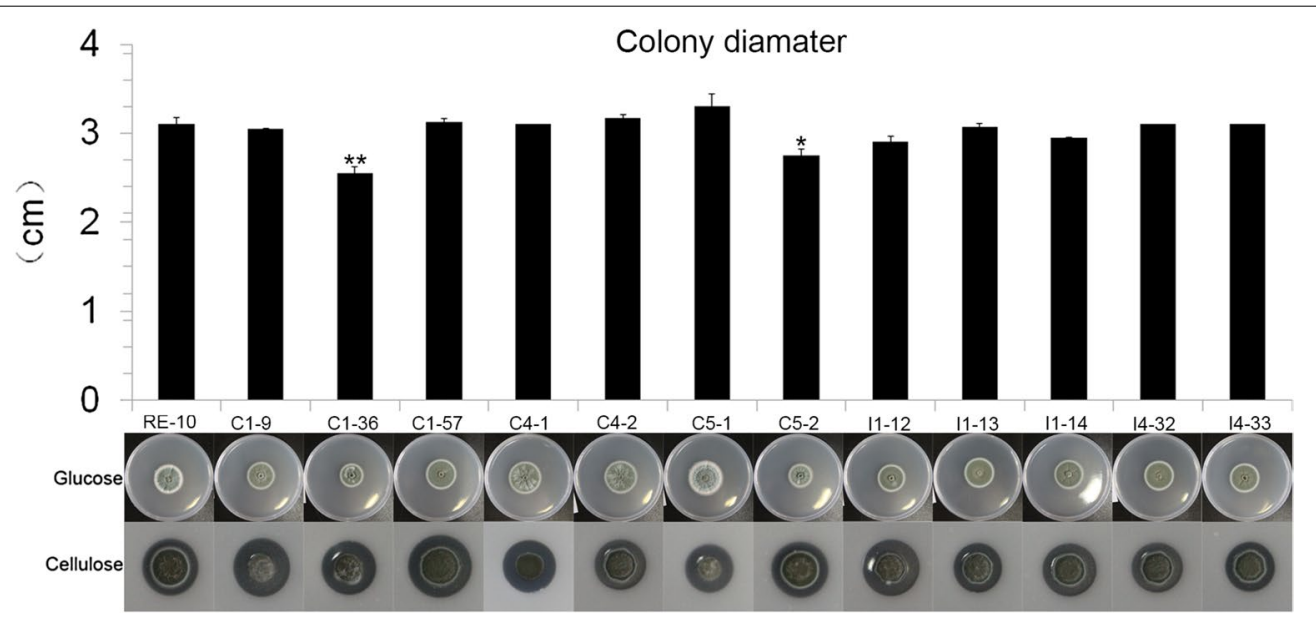

Fig. 3 The effects of $\beta$-glucosidase over-expression on fungal growth and cellulolytic ability. Spores of 12 BGL(X) over-expression mutants and RE-10 were inoculated on the glucose and cellulose plates and cultured for 6 days. The diameters of fungal colonies on glucose were measured and displayed in bar column (up) and cellulolytic zones represented their cellulolytic abilities (down) 


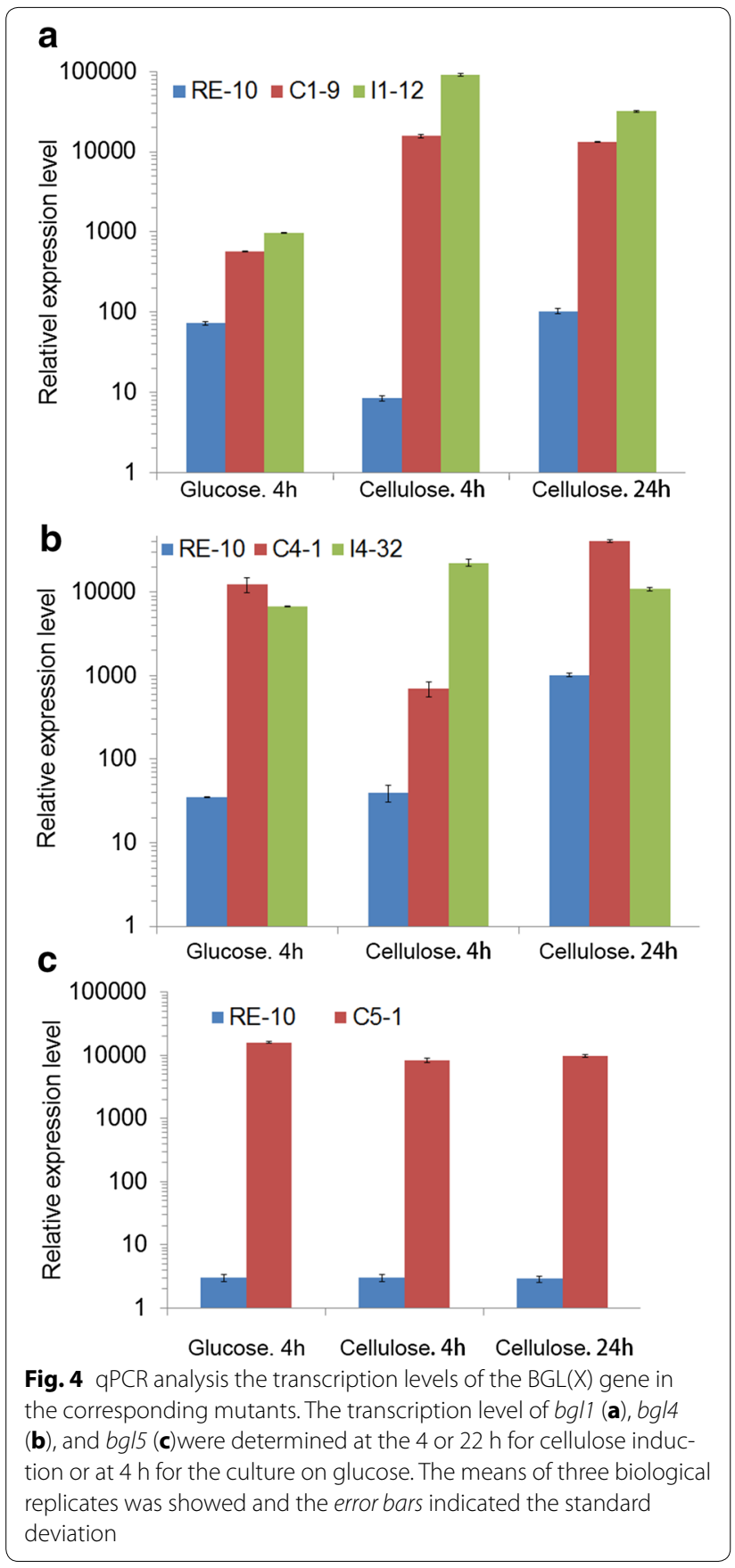

the lignocellulose-glucose conversion. The constitutive $\beta$-glucosidase over-expression strain had a slight higher activity compared to those of corresponding inducible mutants. Especially, C1-9 displayed the highest FPase activity among all with up to $8.0 \mathrm{U} / \mathrm{ml}$ (Fig. 6).

\section{Cellulase enzyme complex with $\beta$-glucosidase over-expression showed higher hydrolysis efficiency against delignified corncob residue (DCCR)}

In nature, neither cellulose, nor filter paper is the staple lignocellulose materials. In order to clarify the hydrolysis ability of the cellulase mixture of all BGL(X) mutants on natural cellulosic materials, the enzyme mixture from their supernatants were used to hydrolyze DCCR. The released glucose for DCCR gradually increased with time from 24 to $72 \mathrm{~h}$. With equal FPU loading, all strains produced similar amounts of glucose from hydrolyzing DCCR, with approximately $40 \mathrm{~g} / \mathrm{l}$ during $72 \mathrm{~h}$. However, we only required $27 \%$ of enzyme loadings for C4-1, $36 \%$ for $\mathrm{C} 5-1,31 \%$ for I1-12, and $38 \%$ for I4-32 to obtain the same hydrolysis rates compared to RE-10. These results signify that these $\beta$-glucosidase over-expression mutants noticeably reduce total crude enzyme loading, and therefore reduce enzyme cost. The amount of released glucose per mg protein is illustrated in Fig. 7. The data clearly shows that all the $\mathrm{BGL}(\mathrm{X})$ mutants produce more than twice or three times the glucose (from 158 to $212 \mathrm{~g}$ glucose/mg crude enzyme complex) at the equivalent protein basis compared to the parental strain RE-10 (65 g glucose/mg crude enzyme complex) (Fig. 7). Although the released glucose of C1-9 was slightly less than that of RE-10 at 72 h, only $22 \%$ protein load was supplemented compared to RE-10. We assume that the mild decrease of hydrolysis rate in C1-9 to be due to a lack of other proteins that play a role in the DCCR hydrolysis. Noteworthy, the strain I4-32 displayed significantly higher glucose yield compared to that of RE-10 at lower protein loading. Engineering the $\beta$-glucosidase as described in this study contributes to manufacture high-efficiency cellulase complex.

A growing number of publications report the reduction of enzyme loadings in the saccharification process and supplementing $\beta$-glucosidase undoubtedly becomes one of the most efficient strategies [22, 30,31]. In this study, the $\beta$-glucosidases were directly improved by strain engineering, which is both time and labor-saving. Lytic polysaccharide monooxygenase (LPMO) [32, 33], expansion and expansion-like proteins [34], and other proteins [35] were reported to play important and synergetic roles in lignocellulose degradation. Their supplementation could significantly improve the lignocellulose-to-glucose conversion rate. With the highly efficient promoters found in this study, we were able to enhance their levels by overexpressing those proteins, and thereby ultimately reduce the enzyme cost. 


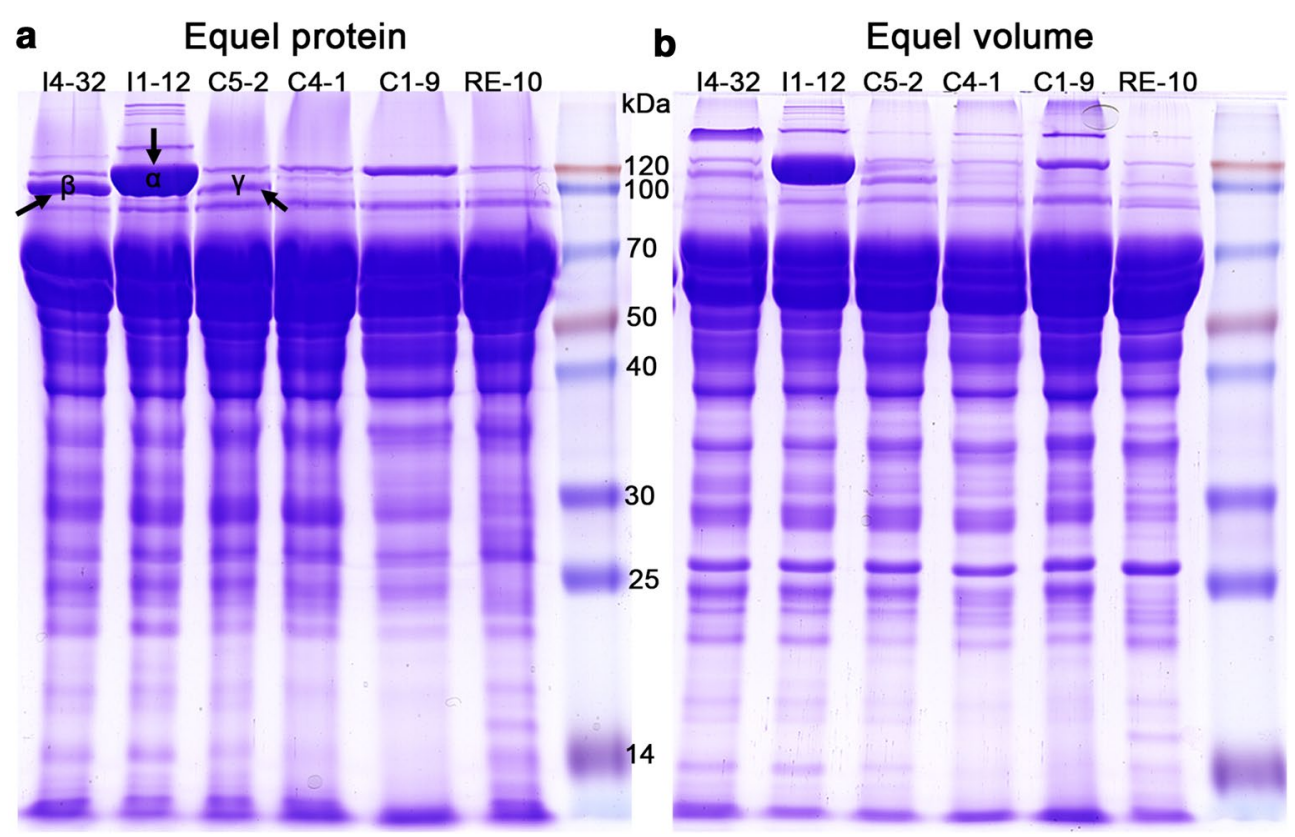

Fig. 5 The increase of $\beta$-glucosidase at the protein level analyzed by SDS-PAGE and mass spectrometry. Equal amount (a) and equal volume (b) of crude enzymes of five BGL(X) over-expression mutants and RE-10 were used for SDS-PAGE analysis and MS identification (a BGL 1; $\beta$ BGL4; $\gamma$ BGL5)

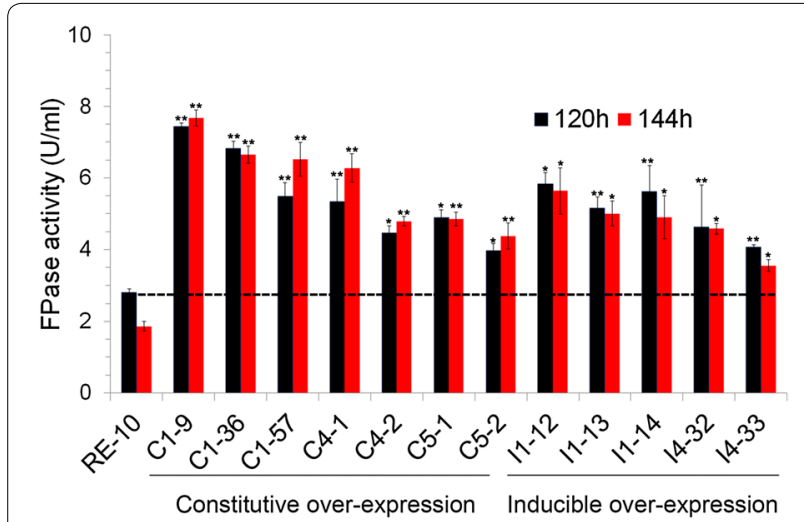

Fig. 6 Analysis of hydrolysis ability of crude enzymes against filter paper. The culture supernatants of the $B G L(X)$ over-expression mutants and the parental strain RE-10 were sampled at 120 and $144 \mathrm{~h}$. The released glucose from filter paper was measured. Data are the means of three biological replicates and error bars show the standard deviation. Asterisks represents the significantly difference between the mutant and the RE-10 ( ${ }^{*} p \leq 0.05$; $^{* *} p \leq 0.01$ )

\section{Conclusions}

This study presents over-expression analysis of nine $\beta$-glucosidase genes in wild-type $P$. oxalicum and identifies three major players of lignocellulose degradation: BGL1, BGL4 and BGL5. Two different types of promoters were used to over-express the three $\beta$-glucosidases in the cellulase over-producer RE-10. Compared to the parental strain, we observed significant boost in beta-glucosidase

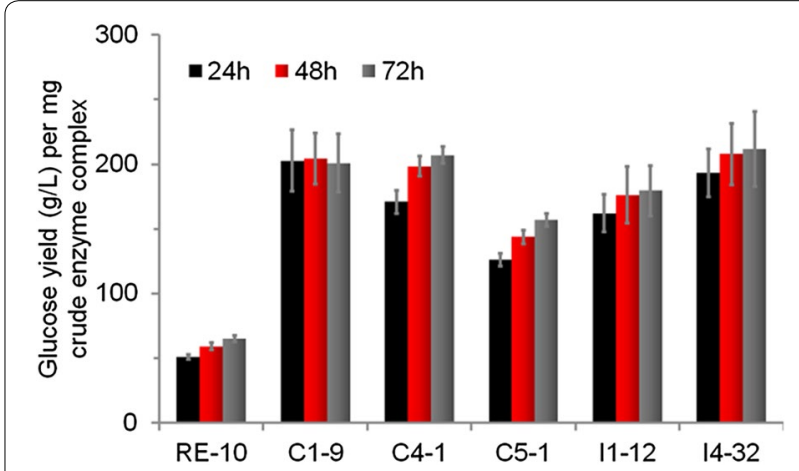

Fig. 7 Saccharification of DCCR with the crude enzymes of $B G L(X)$ over-expression mutants and the parental strain RE-10. The crude enzyme complex from $P$. oxalicum RE-10 and BGL(X) over-expression mutants were used for saccharification of DCCR. Culture supernatants of 10 FPA were mixed with DCCR. Released glucose concentration were measured every $24 \mathrm{~h}$. Data are the means of three biological replicates and error bars show the standard deviation

activity for all recombinant strains obtained. The inducible promoter from gene $b g l 2$ is particularly more efficient in activating the $b g l 1$ or bgl4 gene expression and is proposed to be a potentially valuable tool to conditionally over-express other essential genes in P. oxalicum as well as other cellulolytic fungi. The crude cellulase mixture from these $\beta$-glucosidase over-producers was significantly more efficient in hydrolyzing microcrystalline cellulose, filter paper, and DCCR compared to that 
of RE-10. This substantially reduced the enzyme cost of lignocellulose-based biofuel.

\section{Methods}

\section{Strains and culture conditions}

P. oxalicum 114-2 (CGMCC 5302), the cellulase highproducer RE-10 [13] and all mutants we obtained from these two, were maintained on malt extract agar. The glucose and cellulose medium comprised $1 \times$ Vogel's salts [51] and $2 \%$ glucose or microcrystalline cellulose as the sole source of carbon. The enzyme-producing medium was composed as follows: corn cob residue $(2.0000 \%)$, cellulose $(0.6000 \%)$, wheat bran solid $(4.6571 \%)$, soybean cake powder plate $(1.0000 \%),\left(\mathrm{NH}_{4}\right)_{2} \mathrm{SO}_{4}(0.2000 \%)$, $\mathrm{NaNO}_{3}(0.2789 \%)$, urea (0.1000 \%), $\mathrm{KH}_{2} \mathrm{PO}_{4}(0.3000 \%)$, and $\mathrm{MgSO}_{4}(0.0500 \%)$. To induce enzyme production, $10^{7}$ spores were pre-cultured for $22 \mathrm{~h}$ in glucose medium and subsequently transferred into $1 \times$ Vogle's solution devoid of any carbon source, where it stayed for $2 \mathrm{~h}$. As a final step, the cultures were then transferred to cellulose or enzyme-producing media at $30{ }^{\circ} \mathrm{C}$, and $200 \mathrm{rpm}$ for $144 \mathrm{~h}$. All samples were collected at the respective time points as described in the text.

\section{Bioinformatics analysis}

The sequences of BGL1, BGL4, and BGL5 were used as the search queries in the NCBI database (http://www. ncbi.nlm.nih.gov/). All BGL sequences form $P$. oxalicum and their orthologs from other fungi were downloaded from NCBI's protein database. The software package MEGA 6.0 [36] was used to construct phylogenetic trees with neighbor-joining algorithms and using 500 bootstrap replications. The domain predictions for BGL1, BGL4, and BGL5 were performed using Pfam 28.0 (http://pfam.xfam.org/) and the signal peptide prediction was based on SignalP 3.0 Server (http://www.cbs.dtu.dk/ services/SignalP-3.0/).

\section{Generation of the BGL(X)-over-expression mutant}

Throughout this study, we used high-fidelity FastPfu DNA polymerase (TransGen Biotech, Beijing, China) for all PCR. PEG-mediated fungal transformation protocols were performed following published protocol [37]. The constitutive promoter from gene $p d e_{-} 02864$ was PCRamplified with primers DF and DR, using the P. oxalicum 114-2 genome as the template. We amplified the gene encoding regions of nine $\beta$-glucosidases from $P$. oxalicum 114-2 with $20 \mathrm{bp}$ overlapping fragments including the promoter and the downstream $h p h$ cassette. The resistance cassette of $h p h$ was amplified with primers HPHsF and HPHsR, and via the plasmid $p$ Silent-1 [38] template. These gene over-expression cassettes were fused in the order $\left.p d e_{-} 02864{ }_{(\mathrm{p})}\right)^{-b g l x_{\text {(coding region) }}-h p h \text { by Double-joint }}$
PCR [39] with nest primers DF2 and HPHsR. All the $\mathrm{BGL}(\mathrm{X})$ over-expression cassettes were gel-purified and transformed into the protoplast of P. oxalicum 114-2.

In order to generate the $\operatorname{bgl}(\mathrm{x})$ over-expression mutant in RE-10, the mark gene $h p h$ of the over-expression cassettes of BGL1, BGL4, BGL5 were replaced with a novel resistance gene sur [12] using the primer pair SurF + SurR. The inducible expression cassettes for BGL1 and BGL4 were generated by individually amplifying their encoding region with corresponding primers (see Additional file 6: Table S1) and fused with both the $b g l 2$ promoter and the mark gene sur. All the overexpression cassettes were concentrated by gel purification and transformed into P. oxalicum RE-10 protoplasts.

\section{Beta-glucosidase activity, SDS-PAGE, and HPLC assays}

The fermentation broth was collected via centrifugation, and the aliquots of the supernatant were diluted to measure enzyme activity. To examine $\beta$-glucosidase activity, $p$ NPG and cellobiose (Sigma, USA) were used as substrates. We conducted the enzyme reaction in acetate buffer $\left(\mathrm{pH} \mathrm{4.8)}\right.$ at $50{ }^{\circ} \mathrm{C}$ for a total of $30 \mathrm{~min}$, after which we added $10 \% \mathrm{Na}_{2} \mathrm{CO}_{3}$ to stop the reaction. $p \mathrm{NP}$ release was measured and the absorbance was read at $405 \mathrm{~nm}$. The glucose level was measured with the Biosensor. One enzyme activity unit represents the amount of enzyme required to either produce one $\mu \mathrm{mol}$ glucose, or $p$ NP per minute under the above condition. To measure the FPase activity, the enzyme reaction was conducted in $0.2 \mathrm{~mol} / \mathrm{L}$ acetate buffer $(\mathrm{pH} 4.8)$ at $50{ }^{\circ} \mathrm{C}$ for a total of $60 \mathrm{~min}$ with $0.05 \mathrm{~g}$ Whatman No. 1 paper as the substrate. DNS method was used to quantify the released reducing sugars. A protein concentration assay was performed using a Bradford kit (Sangon Biotech, Shanghai, China). Three biological triplicates were performed throughout all described experiments. Both equal quality and volume of culture supernatants were performed for SDS-PAGE analysis, and the predicted $\beta$-glucosidase bands were excised for MALDI-TOF-MS identification. We measured the released sugars within the broth supernatants via LC-10 AD HPLC (Shimadzu, Japan) by a BioRad Aminex HPX-42A carbohydrate column (Bio-Rad, USA).

\section{Fungal growth, microcrystalline cellulose hydrolysis and $\mathrm{qRT}$-PCR analysis}

Equal volumes of conidia $\left(10^{4}\right.$ per $\left.\mathrm{ml}\right)$ of all $b g l(\mathrm{x})$ mutants as well as the parental strains RE-10 were spotted on a solid plate using $2 \%$ glucose or $3 \%$ ball-milled cellulose at $30{ }^{\circ} \mathrm{C}$ for a total of 6 days, after which, they were photographed. The diameters of all colonies on glucose plates were measured, and this was used to evaluate fungal growth. Two biological triplicates were performed in the analysis. For RNA 
extraction, we inoculated the spores into glucose medium and pre-culturing them for $22 \mathrm{~h}$, followed by a starvation period of $2 \mathrm{~h}$ and transferral into cellulose medium at $30{ }^{\circ} \mathrm{C}$ and $200 \mathrm{rpm}$ for a total of $4 \mathrm{~h}$. The RNAiso ${ }^{\mathrm{TM}}$ reagent and PrimeScript RT Reagent Kit (TaKaRa, Japan) were used to extract RNA and to synthesize cDNA, respectively, following the manufacture's description. We performed the qPCR on LightCycler equipment as previously described [10]. We used the expression level of actin as the internal control for data normalization.

\section{Saccharification of delignified corncob residue}

The delignified corncob residue was provided by LONGLIVE Co., Yucheng, Shandong province, China (http://www.longlive.cn/). Alkaline extraction of the lignin from corncob residues was performed and left the delignified corncob residue. The crude enzymes were collected at $144 \mathrm{~h}$, and we removed the mycelia and residual medium. The saccharification reaction was implemented at $50{ }^{\circ} \mathrm{C}$ in a $5 \mathrm{ml}$ Eppendorf tube containing a mixture of $0.1 \mathrm{~g}$ DCCR (5\%), $1 \% \mathrm{NaN}_{3}$ and $10 \mathrm{FPU} / \mathrm{g}$ glucan per crude enzyme with added $\mathrm{pH} 4.8$ sodium acetate buffer to increase total volume to $2 \mathrm{ml}$. The glucose release was measured using the SBA-40C biological sensor (Shandong, China). The experiments were performed in three biological repetitions.

\section{Statistics}

We performed one-tail $t$-Student tests with equal variance using the software Microsoft Office 2010 Excel. All mean values, standard deviations as well as $p$ values were calculated in the quantitative analyses throughout this study.

\section{Additional files}

Additional file 1: Figure S1. Domain structure of BGL1, BGL4, BGL5.

Additional file 2: Figure S2. Cellobiose hydrolysis activity assays. The culture supernatants of the BGL(X) over-expression mutants and the parental strain RE-10 were sampled at 120 and $144 \mathrm{~h}$. $\beta$-glucosidase activity was measured with the cellobiose as the substrate. Data are the means of three biological replicates and error bars show the standard deviation.

Additional file 3: Figure S3. HPLC assays for the released sugars. The released sugars during the fermentation for all strains were collected, and analyzed by HPLC to quantify the amount of glucose (red) and cellobiose (blue).

Additional file 4: Figure S4. $q P C R$ analysis of the transcription change of cbhland egl The transcript abundance of $c b h$ l (A) and egl (B) under cellulose induction $4 \mathrm{~h}, 24 \mathrm{~h}, 48 \mathrm{~h}$ and $72 \mathrm{~h}$ in mutants and RE-10 were analyzed.

Additional file 5: Figure S5. $q P C R$ analysis of the expression pattern of $b g / 1, b g / 4$, and $b g / 5$ The transcript abundance of $b g / 1, b g / 4$, and $b g / 5$ under glucose $(4 \mathrm{~h})$, no carbon source $(2 \mathrm{~h})$, cellulose $(4 \mathrm{~h})$ conditions in $P$. oxalicum wild type strain 114-2 were analyzed.

Additional file 6: Table S1. Primers used in this study.

\section{Abbreviations}

WT: Wild type; BGL/bgl: ß-Glucosidase; DCCR: delignified corncob residue; Cdt: Cellodextrin transporter; REXRN: reconstruction of expression regulatory network; GH family: glucoside hydrolase family; pNPG: 4-Nitrophenyl- $\beta$-Dglucopyranoside; LPMO: lytic polysaccharide monooxygenase; SDS-PAGE: sodium dodecyl sulfate polyacrylamide gel electrophoresis; HPLC: high-performance liquid chromatography.

\section{Authors' contributions}

GSY conceived the work, analyzed the data, and drafted the manuscript. RMW and QBK performed the experiments and analyzed the data. LWG, ML, JD, and PY participated in the experiment, and collected the data. ZHL design the work. YBQ designed the work and revised the manuscript. All authors read and approved the final manuscript.

\section{Author details}

${ }^{1}$ State Key Laboratory of Microbial Technology, Shandong University, Jinan City 250100, Shandong Province, China. ${ }^{2}$ National Glycoengineering Research Center, Shandong University, Jinan City 250100, Shandong Province, China. ${ }^{3}$ Department of Bioengineering, Qilu University of Technology, Jinan City 250353, Shandong Province, China.

\section{Acknowledgements}

This work is supported by Grants from the National Natural Science Foundation of China (Grant Nos. 31030001, 31370086 and 31200065). ZHL was also supported by grants for China Postdoctoral Science Foundation (2014M561890), Young Excellent Scientists of Shandong Province (Grant no. BS2013SW017), Shandong Province Postdoctoral innovation projects of special funds (Grant no. 201203037), and State Key Laboratory of Microbial Technology Open Projects Fund (No. M2014-07).

\section{Competing interests}

The authors declare that they have no competing interests.

Received: 4 January 2016 Accepted: 18 March 2016

Published online: 31 March 2016

\section{References}

1. Pauly M, Keegstra K. Cell-wall carbohydrates and their modification as a resource for biofuels. Plant J. 2008;54:559-68.

2. Wilson DB. Cellulases and biofuels. Curr Opin Biotechnol. 2009;20:295-9.

3. Merino ST, Cherry J. Progress and challenges in enzyme development for biomass utilization. Adv Biochem Eng Biotechnol. 2007;108:95-120.

4. Percival Zhang YH, Himmel ME, Mielenz JR. Outlook for cellulase improvement: screening and selection strategies. Biotechnol Adv. 2006;24:452-81.

5. Gusakov AV. Alternatives to Trichoderma reesei in biofuel production. Trends Biotechnol. 2011;29:419-25.

6. Liu G, Zhang L, Wei X, Zou G, Qin Y, Ma L, Li J, Zheng H, Wang S, Wang C, et al. Genomic and secretomic analyses reveal unique features of the lignocellulolytic enzyme system of Penicillium decumbens. PLoS ONE. 2013;8:e55185.

7. Liao H, Li S, Wei Z, Shen Q, Xu Y. Insights into high-efficiency lignocellulolytic enzyme production by Penicillium oxalicum GZ-2 induced by a complex substrate. Biotechnol Biofuels. 2014;7:162.

8. Zhao XH, Wang W, Tong B, Zhang SP, Wei DZ. A newly isolated Penicillium oxalicum 16 cellulase with high efficient synergism and high tolerance of monosaccharide. Appl Biochem Biotechnol. 2015;178:173-83.

9. Liu G, Zhang L, Qin Y, Zou G, Li Z, Yan X, Wei X, Chen M, Chen L, Zheng K, et al. Long-term strain improvements accumulate mutations in regulatory elements responsible for hyper-production of cellulolytic enzymes. Sci Rep. 2013;3:1569.

10. Li J, Liu G, Chen M, Li Z, Qin Y, Qu Y. Cellodextrin transporters play important roles in cellulase induction in the cellulolytic fungus Penicillium oxalicum. Appl Microbiol Biotechnol. 2013;97:10479-88.

11. Hu Y, Liu G, Li Z, Qin Y, Qu Y, Song X. G protein-CAMP signaling pathway mediated by $\mathrm{PGA3}$ plays different roles in regulating the expressions of amylases and cellulases in Penicillium decumbens. Fungal Genet Biol. 2013;58-59:62-70. 
12. Li Z, Yao G, Wu R, Gao L, Kan Q, Liu M, Yang P, Liu G, Qin Y, Song X, et al. Synergistic and dose-controlled regulation of cellulase gene expression in Penicillium oxalicum. PLoS Genet. 2015;11:e1005509.

13. Yao G, Li Z, Gao L, Wu R, Kan Q, Liu G, Qu Y. Redesigning the regulatory pathway to enhance cellulase production in Penicillium oxalicum. Biotechnol Biofuels. 2015;8:71.

14. Singhania RR, Patel AK, Sukumaran RK, Larroche C, Pandey A. Role and significance of beta-glucosidases in the hydrolysis of cellulose for bioethanol production. Bioresour Technol. 2013;127:500-7.

15. Henrissat B. A classification of glycosyl hydrolases based on amino acid sequence similarities. Biochem J. 1991;280(Pt 2):309-16.

16. Lombard V, Golaconda Ramulu H, Drula E, Coutinho PM, Henrissat B. The carbohydrate-active enzymes database (CAZy) in 2013. Nucleic Acids Res. 2014;42:D490-5.

17. Bohlin C, Olsen SN, Morant MD, Patkar S, Borch K, Westh P. A comparative study of activity and apparent inhibition of fungal beta-glucosidases. Biotechnol Bioeng. 2010;107:943-52.

18. Chen M, Qin Y, Liu Z, Liu K, Wang F, Qu Y. Isolation and characterization of a beta-glucosidase from Penicillium decumbens and improving hydrolysis of corncob residue by using it as cellulase supplementation. Enzyme Microb Technol. 2010;46:444-9.

19. Chen M, Qin Y, Cao Q, Liu G, Li J, Li Z, Zhao J, Qu Y. Promotion of extracellular lignocellulolytic enzymes production by restraining the intracellular beta-glucosidase in Penicillium decumbens. Bioresour Technol. 2013;137:33-40

20. Znameroski EA, Coradetti ST, Roche CM, Tsai JC, lavarone AT, Cate $\mathrm{JH}$, Glass NL. Induction of lignocellulose-degrading enzymes in Neurospora crassa by cellodextrins. Proc Natl Acad Sci USA. 2012;109:6012-7

21. Suzuki K, Sumitani J, Nam YW, Nishimaki T, Tani S, Wakagi T, Kawaguch T, Fushinobu S. Crystal structures of glycoside hydrolase family 3 betaglucosidase 1 from Aspergillus aculeatus. Biochem J. 2013;452:211-21.

22. Ma L, Zhang J, Zou G, Wang C, Zhou Z. Improvement of cellulase activity in Trichoderma reesei by heterologous expression of a betaglucosidase gene from Penicillium decumbens. Enzyme Microb Technol. 2011;49:366-71.

23. Nitta M, Furukawa T, Shida Y, Mori K, Kuhara S, Morikawa Y, Ogasawara W. A new Zn(II)(2)Cys(6)-type transcription factor BgIR regulates beta-glucosidase expression in Trichoderma reesei. Fungal Genet Biol. 2012;49:388-97

24. Zhou Q, Xu J, Kou Y, Lv X, Zhang X, Zhao G, Zhang W, Chen G, Liu W. Differential involvement of beta-glucosidases from Hypocrea jecorina in rapid induction of cellulase genes by cellulose and cellobiose. Eukaryot Cell. 2012;11:1371-81

25. Sun J, Glass NL. Identification of the CRE-1 cellulolytic regulon in Neurospora crassa. PLoS ONE. 2011;6:e25654.
26. Antonieto AC, dos Santos Castro L, Silva-Rocha R, Persinoti GF, Silva RN Defining the genome-wide role of CRE1 during carbon catabolite repression in Trichoderma reesei using RNA-Seq analysis. Fungal Genet Biol. 2014;73:93-103.

27. Sorensen A, Lubeck M, Lubeck PS, Ahring BK. Fungal beta-glucosidases: a bottleneck in industrial use of lignocellulosic materials. Biomolecules. 2013:3:612-31.

28. Jun H, Guangye H, Daiwen C. Insights into enzyme secretion by filamentous fungi: comparative proteome analysis of Trichoderma reesei grown on different carbon sources. J Proteomics. 2013;89:191-201.

29. Phillips CM, lavarone AT, Marletta MA. Quantitative proteomic approach for cellulose degradation by Neurospora crassa. J Proteome Res. 2011;10:4177-85.

30. Treebupachatsakul T, Shioya K, Nakazawa H, Kawaguchi T, Morikawa Y, Shida Y, Ogasawara W, Okada H. Utilization of recombinant Trichoderma reesei expressing Aspergillus aculeatus beta-glucosidase I (JN11) for a more economical production of ethanol from lignocellulosic biomass. J Biosci Bioeng. 2015;120:657-65.

31. Gao L, Gao F, Zhang D, Zhang C, Wu G, Chen S. Purification and characterization of a new beta-glucosidase from Penicillium piceum and its application in enzymatic degradation of delignified corn stover. Bioresour Technol. 2013;147:658-61.

32. Muller G, Varnai A, Johansen KS, Eijsink VG, Horn SJ. Harnessing the potential of LPMO-containing cellulase cocktails poses new demands on processing conditions. Biotechnol Biofuels. 2015;8:187.

33. Eibinger M, Ganner T, Bubner P, Rosker S, Kracher D, Haltrich D, Ludwig R, Plank H, Nidetzky B. Cellulose surface degradation by a lytic polysaccharide monooxygenase and its effect on cellulase hydrolytic efficiency. J Biol Chem. 2014;289:35929-38.

34. Liu X, Ma Y, Zhang M. Research advances in expansins and expansionlike proteins involved in lignocellulose degradation. Biotechnol Lett. 2015;37:1541-51.

35. Kim IJ, Lee HJ, Choi IG, Kim KH. Synergistic proteins for the enhanced enzymatic hydrolysis of cellulose by cellulase. Appl Microbiol Biotechnol. 2014;98:8469-80

36. Tamura K, Stecher G, Peterson D, Filipski A, Kumar S. MEGA6: Molecular Evolutionary Genetics Analysis version 6.0. Mol Biol Evol. 2013;30:2725-9.

37. Li ZH, Du CM, Zhong YH, Wang TH. Development of a highly efficient gene targeting system allowing rapid genetic manipulations in Penicillium decumbens. Appl Microbiol Biotechnol. 2010;87:1065-76.

38. Nakayashiki H, Hanada S, Nguyen BQ, Kadotani N, Tosa Y, Mayama S. RNA silencing as a tool for exploring gene function in ascomycete fungi. Fungal Genet Biol. 2005;42:275-83.

39. Yu JH, Hamari Z, Han KH, Seo JA, Reyes-Dominguez Y, Scazzocchio C. Double-joint PCR: a PCR-based molecular tool for gene manipulations in filamentous fungi. Fungal Genet Biol. 2004;41:973-81.

\section{Submit your next manuscript to BioMed Central and we will help you at every step:}

- We accept pre-submission inquiries

- Our selector tool helps you to find the most relevant journal

- We provide round the clock customer support

- Convenient online submission

- Thorough peer review

- Inclusion in PubMed and all major indexing services

- Maximum visibility for your research

Submit your manuscript at www.biomedcentral.com/submit
() Biomed Central 